\title{
REFINEMENT OF SDBC BUSINESS PROCESS MODELS USING ISDL
}

\author{
Boris Shishkov \\ Department of Computer Science, University of Twente, 5 Drienerlolaan, Enschede, The Netherlands \\ b.b.shishkov@ewi.utwente.nl \\ Dick Quartel \\ Department of Computer Science, University of Twente, 5 Drienerlolaan, Enschede, The Netherlands \\ d.a.c.quartel@ewi.utwente.nl
}

Keywords: Business process modeling; Refinement; SDBC; ISDL.

\begin{abstract}
Aiming at aligning business process modeling and software specification, the SDBC approach considers a multi-viewpoint modeling where static, dynamic, and data business process aspect models have to be mapped adequately to corresponding static, dynamic, and data software specification aspect models. Next to that, the approach considers also a business process modeling viewpoint which concerns real-life communication and coordination issues, such as meanings, intentions, negotiations, commitments, and obligations. Hence, in order to adequately align communication and dynamic aspect models, SDBC should use at least two modeling techniques. However, the transformation between two techniques unnecessarily complicates the modeling process. Next to that, different techniques use different modeling formalisms whose reflection sometimes causes limitations. For this reason, we explore in the current paper the value which the (modeling) language ISDL could bring to SDBC in the alignment of communication and behavioral (dynamic) business process aspect models; ISDL can usefully refine dynamic process models. Thus, it is feasible to expect that ISDL can complement the SDBC approach, allowing refinement of dynamic business process aspect models, by adding communication and coordination actions. Furthermore, SDBC could benefit from ISDL-related methods assessing whether a realized refinement conforms to the original process model. Our studies in the paper are supported by an illustrative example.
\end{abstract}

\section{INTRODUCTION}

A number of software development approaches have failed because of being insufficiently capable to grasp and utilize the original business information. As argued in (Shishkov \& Dietz, 2004-1), the specification of software and the analysis (and modeling) of its corresponding business processes, should be considered as one integrated task.

The SDBC ('SDBC' stands for Software Derived from Business Components) approach (Shishkov, 2005; Shishkov \& Dietz, 2005) addresses this challenge, by allowing for a sound mapping between a business process model and a software specification model. They both consist of corresponding aspect models which relate to particular viewpoints. Hence, achieving consistency in such a multi-viewpoint modeling is claimed to be crucial (Dijkman, 2006).

SDBC considers three essential modeling viewpoints: Structural viewpoint (about the statics), Behavioral viewpoint (about the dynamics), and Informational viewpoint (about the data).

Further, the approach considers also a business process modeling viewpoint concerning real-life communication/coordination issues: even though software systems are usually well-structured and rules-driven, the business systems which they are to support, are more complex, driven by real-life communication. Thus, the Communication viewpoint plays a role in SDBC, with respect to semantic and pragmatic real-life aspects. Such aspects concern meanings, intentions, negotiations, commitments, and obligations (Shishkov et al., 2006). 
In SDBC, the Communication and Behavioral viewpoints are considered in combination, as complementing each other. For example, it is usual in SDBC that we address (using the DEMO Process Step Model (Dietz, 1999), for instance) the Communication viewpoint, by extending a structural business process aspect model. Then we reflect this in a behavioral business process aspect model (using Petri Nets (Aalst \& Best, 2003), for instance).

Hence, in order to adequately align communication and behavioral aspect models, SDBC should use at least two modeling techniques. However, the transformation between two techniques unnecessarily complicates the modeling process. Next to that, different techniques use different modeling formalisms whose reflection sometimes may cause limitations.

The (modeling) language ISDL (ISDL; Quartel et al., 2005) - 'ISDL' stands for Interaction Systems Design Language, is powerful as concerns the refinement of dynamic process models and corresponding assessment for correctness. Thus, it is feasible to expect that ISDL can usefully complement the SDBC approach, allowing refinement of dynamic business process aspect models, by adding communication and coordination actions.

This has motivated our studying potentials for combining SDBC and an integrated modeling facility based on ISDL. In particular, we explore in this paper the value which ISDL could bring to SDBC in the alignment of communication and dynamic business process aspect models.

The existence of ISDL-related methods allowing assessment whether a refinement conforms to the original process model, further justifies the claim that ISDL can be useful for refining dynamic business process models in SDBC. ISDL could also add value in the SDBC-driven mapping of such models towards software specification, particularly in the context of the design of software services (Quartel et al., 2004), since a mapping mechanisms exist between ISDL and BPEL/WSDL specifications.

The outline of this paper is as follows: Section 2 considers SDBC, paying attention particularly to concepts that concern the Communication and Dynamic viewpoints (discussed before). Then we present in Section 3 an illustrative example to be used in our further studies. On this basis, we discuss in Section 4 how ISDL could support SDBC in the alignment of communication and dynamic business process aspect models. Section 5 analyzes then the value of applying SDBC and ISDL in combination. And finally, Section 6 presents the conclusions.

\section{SDBC}

The approach SDBC supports software design, by providing a business process modeling output that is useful for the specification of the software systemto-be.

Introducing the approach in detail is beyond the scope of this paper; for information about SDBC, interested readers are referred to (Shishkov, 2005).

However, the actual problem which we address (in the paper) is the consistency in aligning communication and dynamic aspect models that concern business process modeling and related software specification. As mentioned before, reaching such a consistency requires appropriate refinement of such models.

That's why, after briefly presenting essential relevant features of SDBC in the following subsection, we will particularly consider, in Sub-section 2.2 , concepts that concern the problem mentioned above.

\subsection{Relevant Features}

Next to the mentioned reference (Shishkov, 2005) to exhaustive information about SDBC, readers could find a general outline of the approach in the current Proceedings (Shishkov et al., 2006). As for SDBC's features that are relevant to the problem addressed in this paper, they will be briefly summarized below, with the help of Figure 1.

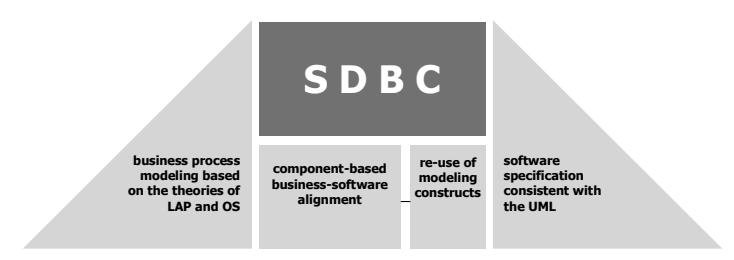

Figure 1: Essential features of SDBC

As seen from the figure, fundamental values of the approach are: 1) its capability of grasping essential real-life aspects, with regard to a business process modeling task, supported by the theories of LAP and OS; 2) its UML-consistent software specification output; 3) its allowing for a sound business-software alignment, driven by the CBD Paradigm; 4) its re-use potentials regarding business/software modeling constructs. These issues will be (briefly) discussed below: 
By applying the Language-Action Perspective LAP (Winograd \& Flores, 1986) and Organizational Semiotics - OS (Liu, 2000), the SDBC approach is capable of capturing the essential aspects (including semantic and pragmatic aspects) that concern an approached business reality - as studied in (Shishkov et al., 2006). SDBC not only provides a modeling framework suitable for such a LAP-OS incorporation but also supports the mapping of a LAP-OS-driven business process modeling output to a software specification model. In this way, the SDBC approach guarantees that the software application-to-be would be adequately operational in the business environment in which it would have to function.

As for its software specification output, SDBC guarantees the consistency of this output with the Unified Modeling Language - UML (Rational, 2005) and other de facto software design standards, supported by a use case derivation procedure (Shishkov \& Dietz, 2004-2). Use case models are derived and then reflected in UML Class diagrams, Activity diagrams, and so on. Hence, such a software specification output is straightforwardly usable by other methods and tools.

The business-software alignment itself (in SDBC) is component-based, founded in the CBD Paradigm - 'CBD' stands for Component-Based Development (Shishkov, 2005). Such an alignment allows for a good traceability between business and software modeling constructs.

Further, the component-based business-software alignment in SDBC allows for re-using modeling constructs. This essentially improves the modeling process since building new models includes re-using previously built modeling constructs.

\subsection{Essential Concepts}

SDBC addresses the Communication viewpoint, by applying the LAP theory which has been mentioned above (readers can find information about it in the current Proceedings (Shishkov et al., 2006)). Crucial in this respect is the SDBC interpretation of the LAP-driven Transaction concept.

The generic process of a Transaction is depicted in Figure 2. If everything goes smoothly, a request is followed by a promise, which is followed by a statement (which is preceded by a noncommunicative production act), which is followed by the acceptance of the production fact. However, an actor could also enter discussions (negotiations). For example, if Mary asks for a pizza, it might happen that the sales person (Paul) says that the shop is closing soon and only hamburgers could be offered - so, this is the discussion, Mary could accept this or not. If she accepts, Paul states a promise regarding this updated request. If she does not like the hamburger, when Paul states it is ready again they enter a discussion (whether another hamburger should be delivered, for example). It is to be noted that, depending on the outcome of such discussions, a Transaction could reach failure and no production fact would then have appeared.

That is why Figure 2 presents three layers. The F-Layer concerns situations in which, as a result of negotiations, a Transaction has reached failure. In such a situation, no production fact has appeared in the business system/environment. The S-Layer concerns situations in which a Transaction has been completed and thus a production fact has appeared. In between these layers is the C-Layer that concerns the process of communication and coordination which determines the ultimate outcome of a Transaction.

Hence, we have four possible communication outcomes, as shown on the figure: 1(2) - agreement is (not) reached and the Executor will (not) realize a production act; 3(4) - the Initiator has (not) accepted the delivered result and a Transaction has (not) appeared.

The three layers discussed above as well as these communication outcomes are of particular importance for the current study. They are depicted on Figure 2, in contrast to a simpler figure (to be found in the current Proceedings (Shishkov et al., 2006)) presenting also the Transaction concept.

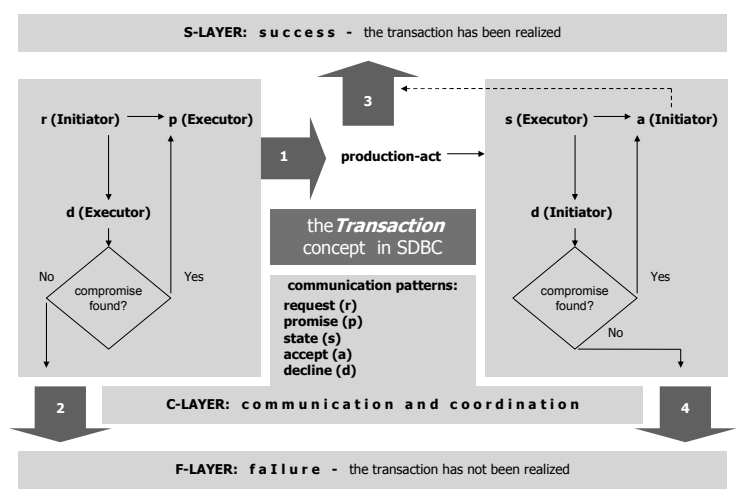

Figure 2: The Transaction concept in SDBC

Therefore, the elementary business process modeling building blocks in SDBC are Transactions. Further, we consider the communication patterns (discussed above), namely: request, promise, state, 
accept, and decline, needed for the elaboration of a Transaction. And finally, by adopting a subjectivist philosophical stance, SDBC acknowledges that nothing exists without a perceiving/acting agent (Liu, 2000). Hence, we need to address the entities related to corresponding Transactions. However, instead of considering the particular agent (entity) involved (human/artificial), SDBC adopts the actorrole (Role) concept (Dietz, 1999). This allows for a sound and flexible modeling - imagine a manager sending a fax, this is not a typical activity for a manager and would therefore make the modeling of such thing complex and confusing, however if we look at this as a role, we could easily model it by introducing the role 'Secretary' (sending a fax is a competence of this role; decision making is a competence of the role 'Manager').

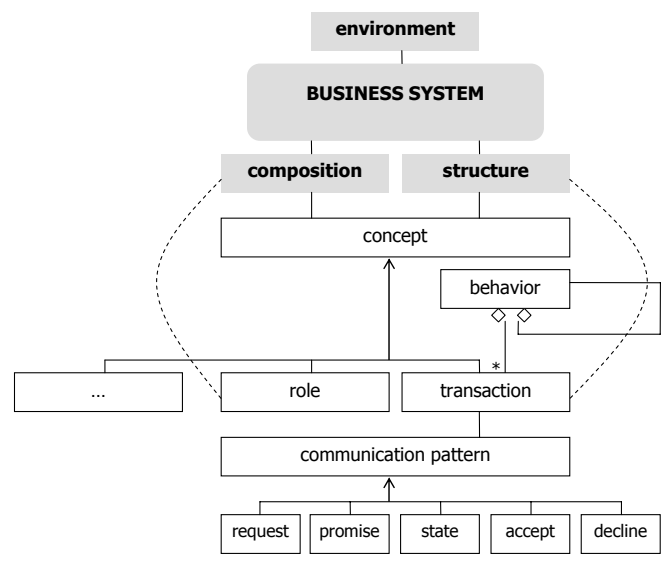

Figure 3: SDBC concepts

We have depicted the mentioned SDBC concepts in Figure 3. In positioning the concepts, we follow the classical views of Bunge (1979), according to which: a (business) system is characterized by composition (it consists of some entities), structure (the entities relate to each other), and environment (entities and relationships outside the system). As seen from the dashed lines, we consider Roles as composition-related concepts, and Transactions as structure-related concepts. The five communication patterns are about the elaboration of a Transaction.

SDBC expresses multi-transaction structures via Petri Nets (Aalst \& Best, 2003), elaborating Transactions via DEMO. This is to be adequately reflected in a UML Activity Diagram.

By applying ISDL here, we expect to reach a simpler and smoother representation, benefiting from ISDL's capability to model and refine a broad range of dynamic patterns (Quartel et al, 2005).
In Section 3, we will introduce an illustrative example and partially approach it with SDBC. Then, ISDL will be introduced and applied to the example, in modeling communication aspects (Section 4). Based on this, we will analyze ISDL's strengths and limitations, in the context of the business-processmodeling-driven specification of software in SDBC.

\section{THE FM EXAMPLE}

The illustrative example that we address in this section, namely the FM example, concerns the Icomp Case. Information about the case can be found in the current Proceedings (Shishkov et al., 2006), and for further elaboration, readers are referred to (Shishkov, 2005).

'FM' stands for Financial Mediator. The FM facilitates insurance companies, in the context of the mentioned case. In order to use the mediator, a company should subscribe (registering for its service).

The support provided by FM to registered companies includes advice and product delivery to their customers:

* a customer can ask FM's advice on which of the companies' products best satisfies a need;

* a customer can also ask FM to deliver a product, on behalf of the particular company.

We will focus on advice delivery only.

To receive advice from FM, the customer should firstly position his(her) request, making it clear whether it is about a health insurance, car insurance, and so on. Secondly, the customer has to specify the particular demand - for instance: to insure a car against theft with the highest possible coverage. Based on this, a Request Processing Unit within the FM generates a standardized specification regarding the customer's request, which is delivered to a Match-making Unit (again within FM). The Matchmaking Unit realizes then a match, supporting in this way the FM in its advice delivery. This match is driven by a particular criterion that is chosen by the customer. For instance: a preference for the cheapest or the most reliable product available. In order to deliver such a criterion-driven match, the Matchmaking Unit uses a data bank that contains relevant rules and procedures. Besides the Request Processing Unit's specification, the Match-making Unit needs as well an input from a Data Search and Processing Unit within FM, in order to realize the match. The Data Search and Processing Unit searches through the information that concerns registered companies, and applies procedures to this 
information. This allows for a precise identification of candidate-matches, relevant to the particular customer's request. Thus, the Match-making Unit puts the candidate-matches list (delivered by the Data Search and Processing Unit) against the standardized request specification (delivered by the Request Processing Unit), and realizes a match, by applying rules and procedures, as above mentioned. All this information, concerning the current example, is partial and we only use it for illustrative purpose.

In applying the SDBC approach to this example, we should start from initial information structuring, identification of role types, and so on (Shishkov, 2005). However, we overlook all initial SDBC analysis and modeling steps and 'arrive' directly at a constructed structural (static) business process aspect model - Figure 4. For more information on these initial modeling steps, readers are referred to (Shishkov, 2005; Shishkov et al., 2006). As for the mentioned model, we have constructed it, using the notations of DEMO (Dietz, 1999). The model concerns the two essential SDBC concepts: Roles and Transactions (as they are depicted in Figure 3).

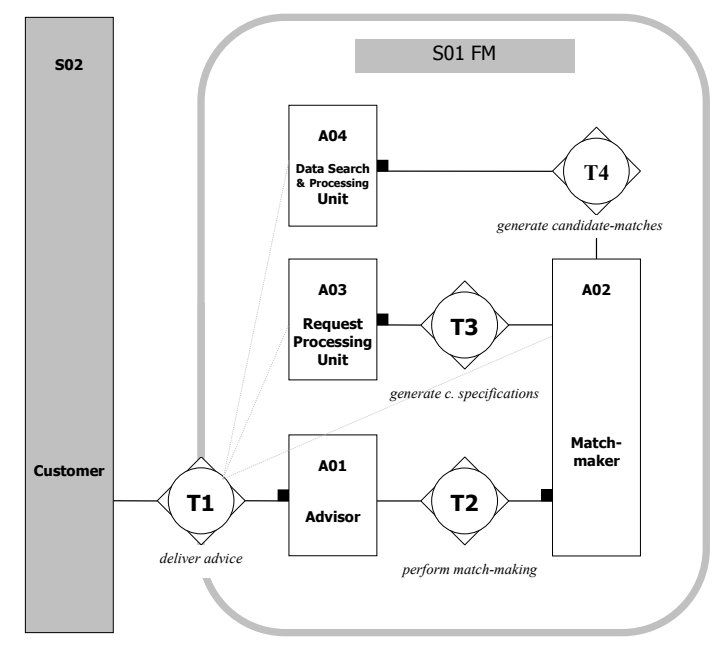

Figure 4: Static (structural) aspect model in SDBC

As seen from Figure 4, an external role type is modeled (Customer) as well as four internal role types (Advisor, Match-Maker, Request Processing Unit, and Data Search and Processing Unit) and four transaction types (Deliver advice; Perform matchmaking; Generate customer's information specification; Generate candidate-matches). The rounded rectangle indicates the boundary of our system. The black boxes indicate which role holds the responsibility for a Transaction.
Now our task is to proceed to communication and dynamic aspect modeling. This is to include elaboration of the modeled Transactions in terms of communicative acts and coordination (staying consistent with the Transaction notion - Figure 2), and also modeling of the flows of production facts.

This is addressed in Section 4. It will explore the value which ISDL could bring to SDBC. As mentioned, we envision potentials for a useful ISDL support to SDBC in the alignment of communication and dynamic business process aspect models.

\section{APPLYING ISDL}

The strengths of ISDL, which are envisioned with regard to the considered research challenges, will be demonstrated in this section, after we briefly introduce the language.

ISDL is aimed at system modeling at higher abstraction levels. In particular, the language is used for business process modeling and application design (Quartel et al., 2005; Quartel et al., 1997).

ISDL provides a small, but expressive set of basic and generic concepts for behavior modeling, aimed at modeling the behavior of systems from varying domains and at successive abstraction levels. The semantics of ISDL has been defined formally, and a method for conformance assessment has been defined. Furthermore, an integrated editor and simulator is available, and tools supporting conformance assessment and model-to-model (code) transformations are being developed.

\subsection{Concepts and Notation}

Figure 5 depicts part of the behavior conceptual model of ISDL, including the entity concept. Figure 6 shows how these concepts are represented.

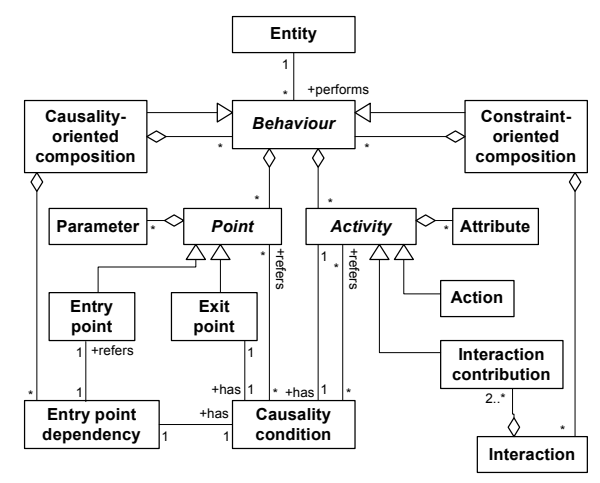

Figure 5: ISDL concepts 
The entity concept represents a system part that can perform some behavior. A behavior is essentially a set of causally related activities. An activity represents some unit of behavior that is atomic, i.e., cannot be split at the abstraction level at which it is defined. Further, an activity either happens, in which case reference can be made to its result, or does not happen at all, in which case no reference can be made to any result, not even to partial results. We distinguish three types of activities. An action is performed by a single behavior (entity). Actions are graphically expressed by ovals (or circles). An interaction is performed by two or more behaviors in cooperation. An interaction is expressed as two or more connected interaction contributions which represent the participation of the involved behaviors. Interaction contributions are expressed by oval (or circle) segments.

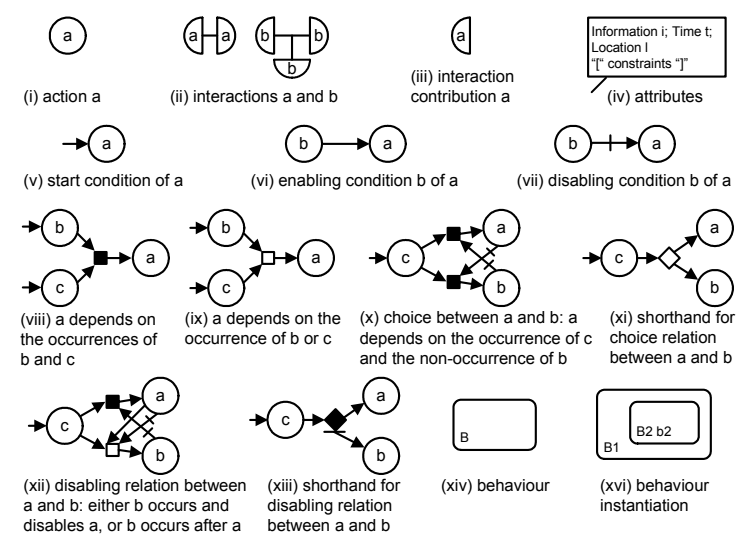

Figure 6: ISDL language elements

An activity can have attributes to represent the relevant characteristics of the occurrence of the modeled real-world activity. Predefined attributes are the information, time and location attribute (see Figure 6 (iv)), representing the activity result (e.g., some information or product), the time of occurrence at which the result is available, and the location where the result is available, respectively. Constraints can be defined on the possible attribute values. The constraints also specify the relation between attribute values established in causally dependent activities. ISDL does not prescribe a language for defining attribute types and constraints, but provides bindings to existing languages that can be used for that purpose. Currently, bindings to $Z$, Java and $Q$ exist.

Relations between activities are modeled by causality conditions. Each activity has a causality condition, which defines how this activity causally depends on other activities. An activity is enabled, i.e., allowed to occur, if its causality condition is satisfied. Three types of basic causality conditions are identified as illustrated in Figure 6: (v) the start condition represents that activity a is enabled from the beginning of some behavior and independent of any other activity, (vi) enabling condition b represents that activity $b$ must have occurred before a can occur, and (vii) disabling condition $\neg \mathrm{b}$ represents that activity $b$ must not have occurred before nor simultaneously with a to enable the occurrence of $b$. These elementary conditions can be combined using the and- and or-operator to represent more complex conditions. Figure 6 depicts also some simple examples.

Containment of one behavior by another (the composite), is represented by behavior instantiation. A behavior instantiation represents that some behavior instance is created in the context of the behavior that contains the instantiation.

\subsection{Activity Refinement}

An activity cannot be split at the abstraction level at which it is considered. A more detailed model of an activity can be obtained by decomposing this activity into multiple sub-activities and their relationships. The relevant characteristics of these sub-activities can be modeled again by the activity concept (i.e., actions, interactions or interaction contributions). Therefore, the activity concept is independent of the abstraction level or granularity at which specific activities are modeled.

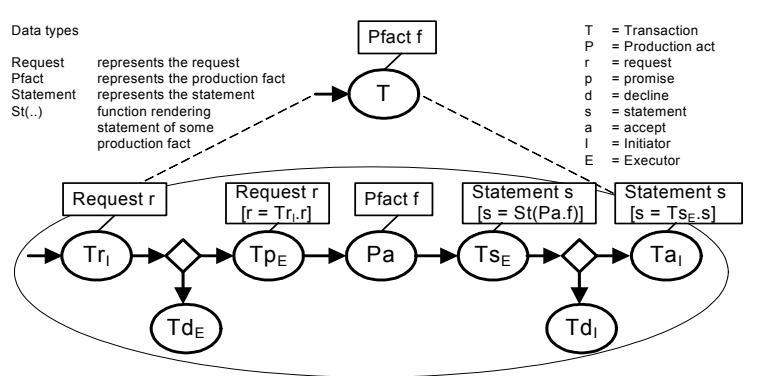

Figure 7: The ISDL Transaction models

In the context of SDBC, the activity concept is used to model Transactions as well their corresponding communication patterns. Figure 7 reflects the generic process of a Transaction, is modeled at two different abstraction levels. At the highest level, the Transaction is represented by a 
single action, which models the production fact that is established. Characteristics of the production fact are modeled using action attributes. At a lower abstraction level, the Transaction's communication aspects are modeled, conforming to Figure 2 . Separate actions are used to model the Transaction's request, promise, state, accept, and decline, and the production act. Observe that actions $\mathrm{Td}_{\mathrm{E}}$ and $\mathrm{Td}_{\mathrm{I}}$ correspond to the decline of a Transaction followed by an unsuccessful negotiation (see Figure 2); and actions $\mathrm{Tp}_{\mathrm{E}}$ and $\mathrm{Ta}_{1}$ represent the promise and acceptance, respectively, which are possibly preceded by an 'initial decline' followed by a successful negotiation.

The result of the Transaction behavior at the lower abstraction level should conform to the result of the Transaction as modeled at the higher abstraction level. In this case, the result of the Transaction behavior is either the occurrence of action $\mathrm{Ta}$, which corresponds to the occurrence of $\mathrm{T}$, or the occurrence of $\mathrm{Td}_{\mathrm{E}}$ or $\mathrm{Td}$, which corresponds to the non-occurrence of T. Consequently, to assess conformance one should assess whether the results as modeled by actions $T a_{1}$ and $T$ are equivalent.

A method has been defined for ISDL to assess the conformance of any abstract behavior to a concrete behavior that refines the abstract behavior. The example in Figure 8 represents a special case of this method. For a detailed explanation of the method, refer to (Quartel et al, 2002).

(i)
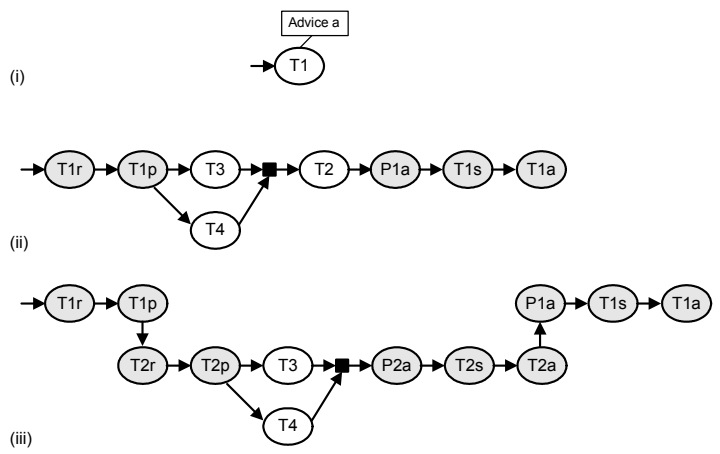

Figure 8: ISDL models of the FM behavior

\subsection{Modeling the FM Example}

Using the ISDL Transaction models presented in Sub-section 4.2, Figure 8 depicts the modeling of the FM example (Section 3) at three successive abstraction levels. At level (ii), some detail is added on how the advice is delivered, eliciting part of the internal behavior of the FM: in this case the communication aspects of $\mathrm{T} 1$ and the use of Transactions T2, T3 and T4. More detail is added in (iii), by elaborating the communication aspects of T2. A similar elaboration can be made for T3 and $\mathrm{T} 4$, but has been omitted for brevity. For the same reason, action attributes are not modeled and it is assumed that Transactions will not be declined. To clearly distinguish between the abstraction levels at which a Transaction is modeled, the communication patterns of a Transaction are indicated in grey.

The ISDL models presented so far do not consider the Roles involved in each Transaction. This aspect can be modeled explicitly using the interaction concept. For example, Figure 9 (i) models Transaction $\mathrm{T} 1$ as an interaction between the role type Customer and FM, where Roles are represented by ISDL behaviors. The interaction concept allows one to model the constraints each Role may have on the possible results (production facts) of the interaction. For example, a customer may restrict the advices (s)he is interested in to car insurances, whereas FM may only consider insurances from particular companies.

(i)
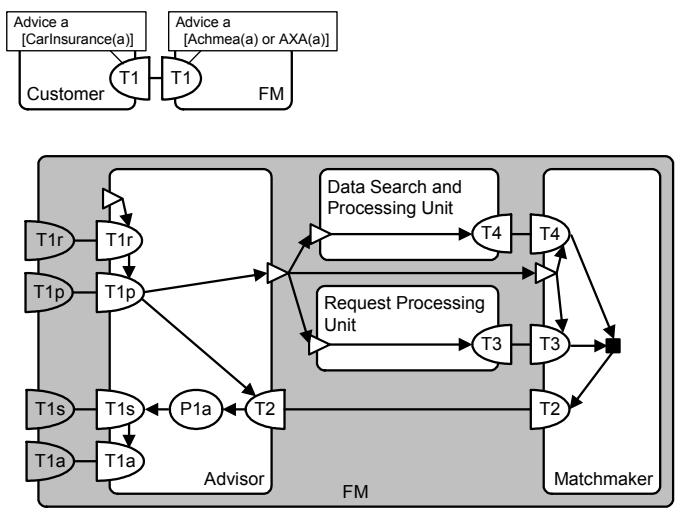

Figure 9: ISDL models of the FM behavior

Figure 9 (ii) presents the ISDL model corresponding to the SDBC model depicted in Figure 4; it is elicited which Roles are involved in which Transactions. In this case the behavior of FM is represented as a composite behavior (indicated in grey). Behaviors in a composite behavior can be related using: (i) constraint-oriented composition: interactions that relate the interaction contributions of the component behaviors; and/or (ii) causalityoriented composition: entry and exit points that represent a causality condition entering a behavior or a causality condition exiting a behavior, respectively. The condition that an entry point represents is associated to it via an entry point 
dependency. Entry and exit points are represented by triangles that point into or out of a behavior, respectively. Interaction contributions of a component behavior can contribute to interactions of their composite behavior. This is represented by drawing a line between the interaction contributions of the component and interaction contributions of the composite (having the same name in the example).

\section{ANALYSIS}

As stated in Section 1, our study on the SDBC-ISDL combination is to be the basis for an analysis (to be conducted in the current section) on the suitability and adequacy of combining the SDBC approach and the ISDL language.

Our fundamental conclusion is that the essential value of combining SDBC and ISDL concerns the possibility to adequately grasp (driven by SDBC) a business reality (addressing not only structural, dynamic, and informational but also communication aspects) and map this towards software specification, facilitated by a powerful language instrumentarium (ISDL), which allows one to align communication and dynamic aspect views, and also add precision in the reflection towards software specification - particularly in the context of service design (Quartel, 2005). Applying the powerful graphical notations of ISDL makes it possible therefore to combine communication and dynamic aspect models, presenting them in a coherent whole (and expressing complex behavior patterns) - ISDL can be used at different abstraction levels and its method for conformance assessment allows one to relate successive abstraction levels. In all this, only a single formalism is needed.

As we have studied, the concepts of ISDL (such as the activity concept) correspond naturally to the behavioral concepts of SDBC (such as the Transaction concept), i.e., ISDL can represent the properties modeled by SDBC concepts. Thus, one can smoothly apply ISDL in the context of the SDBC approach.

Further, complementing SDBC by ISDL, allows for a consideration of the notions of Role and Transaction - these are essential for a business process modeling driven by SDBC (Shishkov \& Dietz, 2005). The interaction concept in ISDL allows one to model transactions between different roles. Furthermore, the transactions, modeled in such a way, can be defined at a high level of abstraction in contrast to e.g. modeling languages using message passing as the basic interaction concept. When using message passing, one is often forced to define transactions closer to implementation level, since one may need multiple messages to exchange the information that is required to establish the desired transaction result. Instead, the interaction concept in ISDL allows each involved role to define its constraints on the possible interaction result, while abstracting from how these constraints are implemented (e.g. through message exchange).

This strong point of ISDL can add value in the context of the SDBC approach. It allows one to decompose a transaction into transaction contributions, defining the responsibility of each role in the transaction (but still at an abstract level). When defining a transaction as an action, one abstracts from the contribution/responsibility of each role in the transaction.

And finally, ISDL can be used to represent both business process concepts and software application concepts (Quartel et al., 2005); nevertheless, the mapping towards software specification is beyond the scope of this paper. Anyway, a mapping/transformation is (being) defined from ISDL to BPEL/WSDL, which should facilitate the implementation of business process models using the Service-Oriented Paradigm (Dirgahayu, 2005).

\section{CONCLUSIONS}

In this paper, we report studies that concern the actual challenge of achieving an adequate alignment between the development of software and the capturing of the business environment in which this software would have to operate. In particular, we have considered the SDBC approach which is capable of realizing such an alignment, by mapping business process aspect models to software specification aspect models. Nevertheless, achieving consistency in such a multi-viewpoint modeling is believed to be crucial. This holds not only for the business-software alignment task but also for the business process modeling itself. It is critical that all business process aspect models in SDBC are consistent with each other.

The SDBC-driven business process modeling concerns not only static, dynamic, and data business process aspect models (as in many current methods) but also a business process modeling viewpoint which is about real-life communication and coordination issues, such as meanings, intentions, negotiations, commitments, and obligations. Thus, it is essential that the SDBC approach allows for a sound alignment between communication and 
dynamic business process aspect models. However, currently the approach uses at least two modeling techniques in order to achieve such an alignment. The transformation among two techniques unnecessarily complicates the modeling process and causes limitations.

Therefore, if we apply within SDBC an integrated language facility based on one formalism and possessing powerful modeling expressiveness, we could improve the alignment between communication and dynamic business process aspect models.

We have identified ISDL as a good candidate in the mentioned context, given its refinement and conformance assessment capabilities as well as powerful graphical notations. In the course of the current study, we have justified this choice, by finding evidence of particular relevant strengths of ISDL. Next to that, we have demonstrated those strengths (and the value of the SDBC-ISDL combination), by means of an illustrative example.

The powerful graphical notations of ISDL, driven by one formalism, proved to work usefully in the context of the SDBC approach. The mentioned notations can support the approach in the alignment of communication and dynamic aspect models, presenting them in a coherent whole. Next to that, ISDL could be used for refinement at different abstraction levels, as demonstrated in Section 4, supported by mechanisms allowing assessment whether a refinement conforms to the original process model. And finally, with regard to serviceoriented platforms, ISDL could support the businesssoftware alignment in SDBC (we have not studied this issue in the current paper), applying an existing mapping facility between ISDL and BPEL/WSDL.

To further this research, we plan to conduct a real-life case study in order to bring practical evidence in support of our findings. Next to that, we will explore further the SDBC-ISDL combination, particularly from the perspective of aligning static and dynamic business process aspect models, and we will study possibilities for simulation-driven validation in SDBC of ISDL models.

\section{ACKNOWLEDGEMENTS}

This work is part of the Freeband AWARENESS and A-MUSE projects (http://awareness.freeband.nl; http://a-muse.freeband.nl). Freeband is sponsored by the Dutch government under contract BSIK 03025.

\section{REFERENCES}

Aalst, W.V.D. and E. Best, 2003. Applications and theory of Petri Nets, Springer, LNCS 2679.

Bunge, M.A., 1979. A world of systems, Treatise on basic philosophy, Vol. 4, Reidel Publ. Company. Dordrecht.

Dietz, J.L.G., 1999. Understanding and modeling business processes with DEMO. In ER'99, $18^{\text {th }}$ International Conference on Conceptual Modeling. Springer LNCS 1728.

Dijkman, R., 2006. Consistency in multi-viewpoint architectural design, Universal Press. Enschede.

Dirgahayu, T., 2005. Model-driven engineering of web service compositions: a transformation from ISDL to $B P E L$, University of Twente - UT Press. Enschede.

ISDL home, http://isdl.ctit.utwente.nl, n.d.

Liu, K., 2000. Semiotics in information systems engineering, Cambridge University Press. Cambridge.

Quartel, D.; R. Dijkman; M. van Sinderen, 2005. An approach to relate business and application services using ISDL. In EDOC'05, $9^{\text {th }}$ IEEE International EDOC Enterprise Computing Conference.

Quartel, D.; R. Dijkman; M. van Sinderen, 2004. Methodological support for service-oriented design with ISDL. In $2^{\text {nd }}$ International Conference on Service Oriented Computing.

Quartel, D.; L. Ferreira Pires; M. van Sinderen, 2002. On architectural support for behaviour refinement in distributed systems design. In Journal of Integrated Design and Process Science, 6(1).

Quartel, D.; L. Ferreira Pires; M. van Sinderen; H.M. Franken, 1997. On the role of basic design concepts in behaviour structuring. In Computer Networks and ISDN Systems.

Rational, 2005. UML Resource Center (http://www.rational.com). USA.

Shishkov, B., 2005. Software specification based on reusable business components, Sieca Repro. Delft.

Shishkov, B.; J.L.G. Dietz; K. Liu, 2006. Bridging the Language-Action Perspective and Organizational Semiotics in SDBC. In ICEIS'06, $8^{\text {th }}$ Int. Conference on Enterprise Information Systems. ICEIS Press.

Shishkov B. and J.L.G. Dietz, 2005. Applying componentbased UML-driven conceptual modeling in SDBC. In ICEIS'05, $7^{\text {th }}$ International Conference on Enterprise Information Systems. ICEIS Press.

Shishkov B. and J.L.G. Dietz, 2004-1. Aligning business process modeling and software specification in a component-based way, the advantages of SDBC. In ICEIS'04, $6^{\text {th }}$ International Conference on Enterprise Information Systems. ICEIS Press.

Shishkov B. and J.L.G. Dietz, 2004-2. Deriving Use Cases from business processes, The advantages of DEMO. Enterprise Information Systems V, Ed: O. Camp, J.B.L. Filipe, S. Hammoudi, M. Piattini, Kluwer Academic Publisher. Dordrecht/Boston/London.

Winograd, T. and F. Flores, 1986. Understanding computers and cognition: a foundation for design, Ablex, Norwood, NJ. 